\title{
El tratamiento antituberculoso intensificado en adultos con meningitis tuberculosa no reduce la mortalidad
}

Intensified antituberculosis treatment in adults with tuberculous meningitis does not reduce mortality

Heemskerk AD y col. N Engl J Med. 2016;374(2):124-34

\section{Objetivos}

Evaluar la mortalidad de un régimen antituberculoso estándar comparado con un régimen intensificado con una dosis más alta de rifampicina y levofloxacina durante las primeras 8 semanas de tratamiento.

\section{Diseño, lugar y pacientes}

Ensayo clínico aleatorizado, doble ciego, controlado con placebo realizado en dos centros de Ho Chi Minh City, Vietnam, en el que se aleatorizaron 817 pacientes adultos $(69 \%$ hombres; mediana de edad, 35 años) con meningitis tuberculosa a uno de dos regímenes.

\section{Intervenciones}

Tratamiento estándar de 9 meses $(n=409)$ : isoniacida, rifampicina $(10 \mathrm{mg} / \mathrm{kg} / \mathrm{día})$, pirazinamida y etambutol durante 3 meses, luego solo isoniacida y rifampicina durante los siguientes 6 meses. Tratamiento intensificado $(n=408)$ : tratamiento estándar más dosis altas de rifampicina (15 mg/ $/ \mathrm{kg} /$ día) y levofloxacina (20 mg/kg/día) durante las primeras 8 semanas, luego solo el tratamiento estándar. Todos los pacientes recibieron tratamiento con dexametasona durante las primeras 6 a 8 semanas.

\section{Medición de resultados principales}

El resultado primario fue la mortalidad a 9 meses medida por intención de tratar. Los resultados secundarios fueron discapacidad neurológica, el tiempo al primer nuevo evento neurológico y eventos adversos serios.

\section{Resultados Principales}

A 9 meses de seguimiento la mortalidad fue casi idéntica en los grupos de tratamiento estándar (113 pacientes) e intensificado (114 pacientes): HR 0,94 (IC 95\% 0,73 a 1,22). Los factores asociados con la mala supervivencia incluyeron los hallazgos neurológicos graves al inicio del tratamiento, la infección por HIV y la infección resistente a múltiples fármacos o a rifampicina. Las alergias, los eventos adversos y las interrupciones del tratamiento fueron más comunes con el tratamiento intensificado, pero las diferencias no eran estadísticamente significativas.

\section{Conclusiones}

El tratamiento intensificado de la meningitis tuberculosa no mejoró la supervivencia en comparación con el tratamiento estándar.

Fuente de financiamiento/conflictos de interés de los autores: Financiado por el Wellcome Trust y la Fundación Li Ka Shing. Los autores no reportaron conflictos de interés.

\section{Comentario}

Desde la introducción del tratamiento antibiótico con estreptomicina en 1950 ha disminuido letalidad de la meningitis tuberculosa, sin embargo, su morbimortalidad sigue siendo alta ${ }^{1}$. La meningitis es la forma más grave de tuberculosis, resultando en muerte o discapacidad neurológica en el $50 \%$ de los pacientes ${ }^{2,3}$.

Las guías de tratamiento actuales para la tuberculosis pulmonar recomiendan un tratamiento en la fase intensiva con rifampicina (10 mg/kg, máximo $750 \mathrm{mg} /$ día), isoniazida $(5 \mathrm{mg} / \mathrm{kg}$, máximo 300 $\mathrm{mg} / \mathrm{día}$ ), pirazinamida (25 mg/kg, máximo $2 \mathrm{~g} /$ día) y estreptomicina (20 mg $/ \mathrm{kg}$, máximo $1 \mathrm{~g} / \mathrm{día}$ ) durante 2 meses; seguido por rifampicina e isoniazida por 7 meses (fase de consolidación). El tratamiento en pacientes con meningitis tuberculosa sigue el modelo de quimioterapia pulmonar, pero el régimen de medicamentos y la duración óptimos no se han establecido ${ }^{2-4}$. La penetración al sistema nervioso central de los diferentes medicamentos antituberculosos de primera línea es baja, en particular, la de rifampicina y etambutol. Se estima que la penetración de la rifampicina al sistema nervioso central es de 10 a $20 \%{ }^{2,3}$. Sumado a esta desventaja, esta droga presenta mucha variación en sus niveles interindividuales debido a sus múltiples interacciones en el metabolismo hepático'.

En un estudio realizado en el año 2013 la mortalidad a los seis meses fue sustancialmente menor en los pacientes con meningitis tuberculosa que recibieron rifampicina en dosis altas por vía intravenosa. Dado que en ese estudio también se había incorporado el uso quinolonas endovenosas, no se puede saber si fue la rifampicina, la moxifloxacina o la sinergia entre ambas drogas las responsables del efecto beneficioso ${ }^{2}$.

Conclusiones del comentador

Los tratamientos actuales de la meningitis tuberculosa se basan en recomendaciones para tratamiento de las formas pulmonares, pero las dosis y la duración de la terapia no están definidas. Se requieren más estudios para evaluar si dosis mayores de rifampicina, probablemente administradas por vía endovenosa y asociadas o no al uso de quinolonas, presentan mejores resultados clínicos.

Astrid Smud [ Sección Infectología, Servicio se Clínica Médica, Hospital Italiano de Buenos Aires. astrid.smud@hospitalitaliano.org.ar ]

Smud A. El tratamiento antituberculoso intensificado en adultos con meningitis tuberculosa no reduce la mortalidad. Evid Actual Pract Ambul. 2018;21(4):105. Comentado de: Heemskerk AD y col. Intensified Antituberculosis Therapy in Adults with Tuberculous Meningitis. N Engl J Med. 2016;374(2):124-34 PMID 26760084.

\section{Referencias:}

1. Heemskerk D y col. Intensified treatment with high dose rifampicin and levofloxacin compared to standard treatment for adult patients with tuberculous meningitis (TBM-IT): protocol for a randomized controlled trial. Trials. 2011;12:25

2. Ruslami $\mathrm{R}$ y col. Intensified regimen containing rifampicin and moxifloxacin for tuberculous meningitis: an open-label, randomised controlled phase 2 trial. Lancet Infect Dis. 2013 Jan;13(1):27-35.

3. Te Brake $\mathrm{L}$ y col. Pharmacokinetic/pharmacodynamic analysis of an intensified regimen containing rifampicin and moxifloxacin for tuberculous meningitis. Int $\mathrm{J}$ Antimicrob Agents. 2015 May;45(5):496-503

4. Nahid P y col. Official American Thoracic Society/Centers for Disease Control and Prevention/Infectious Diseases Society of America Clinical Practice Guidelines: Treatment of Drug-SusceptibleTuberculosis. Clin Infect Dis. 2016 Oct 1;63(7):e147-e195 